\title{
Development of a Novel Multi-Agent System for Residential Voltage Control Using Demand Response based on Customer Behaviour
}

\author{
Sima Davarzani, Ioana Pisica, Gareth Taylor \\ Smart Power Networks, Institute of Energy Futures \\ Brunel University London, UK \\ Sima.davarzani@brunel.ac.uk
}

\begin{abstract}
Aggregation of demand during peak load periods can cause local overloading in distribution networks. Quantifying the available peak demand reduction at a local level enables flexible and dynamic management of variable power flows on a distribution network. Since domestic loads coupled with distributed generation are intermittent and uncertain in nature, a detailed analysis of domestic load profiles is an essential step to achieve flexible demand response. This paper introduces and develops a novel multiagent system approach to provide domestic demand response over time. This new approach is based on customer elasticity when considering different parameters including social, technical and financial aspects of customer behaviour .
\end{abstract}

Keywords -Demand response, multi-agent system, domestic load profile, voltage control

\section{INTRODUCTION}

In recent years, Demand Response (DR) has been introduced as an effective tool in order to regulate voltage in normal and emergency conditions at distribution feeders [1]. Maintaining the power balance equilibrium using DR mechanisms reduces power loss and improves the voltage profile at distribution feeders. However, implementing DR in residential levels is more complex due to intermittent nature and low demand curtailment available from each individual connected load within a feeder.

Multi-Agent Systems (MAS) are the most common tool for modeling a complex systems in analyzing the interaction between multiple autonomous and intelligent components called agents [2]. A number of DR control algorithms have been proposed for residential customers using MAS structure. These studies aim to enhance the system flexibility based on the engagement of various controllable home appliances in DR programs [3-6]. A Distribution Transformer (DT) management algorithm with MAS has been discussed in [7]. The proposed DR algorithm resulted in more than $50 \%$ reduction for an emergency two-hour DR event. In [8], a unified MAS approach has been presented in order to control thermal overloading of the network through local voltage control. However, these papers have not considered the behaviour characterisation of residential customers which highly impact on power consumption patterns and estimating potential of demand reduction during DR event.

This paper presents and develops a novel MAS framework that enables local overloading management for distribution networks by providing DR services from domestic loads. The main objective is to provide an efficient voltage control in the distribution feeders by reducing total active power at the DT during emergency conditions. The proposed methodology is based on estimation and prediction of the potential DR which are available from aggregation of all connected loads within a feeder. In case of voltage drop, a Demand Curtailment Level (DCL) dynamically allocates to a set of customers of different classes. In this regard, a characteristic-based clustering applies to time-series historical residential load profiles, taking into consideration, willingness to engage in DR programs and potential of demand responsiveness during DR event in each cluster. This approach enables a dynamic, active and flexible DR management system considering available elastic peak demand from large scale domestic loads aggregation.

The rest of the paper is structured as follows. Section II discusses the proposed MAS framework and objectives. Moreover, the DR algorithm and accordingly MAS agent's behaviours is described. Methodology of the proposed approach and data initialization are introduced in section III followed by the description of the related case study in section IV. Simulation results are analyzed and discussed in details in Section V and finally the conclusion and future work are drawn in Section VI.

\section{PROPOSED MAS APPROACH}

\section{A. MAS Framework}

In order to model the elasticity of domestic demand responsiveness in this paper, a new multi-layer environment has been developed. The aim of proposed MAS framework is to provide a local energy management based on aggregation of residential customers connected to 
a DT inside a feeder. Toward this scope, the MAS structure consists of four different kinds of DR participants which are individually modeled with an agent as presented in Fig. 1. The modelling details of each agent are presented henceforth.

- Data Communication Company Agent (DCCA): Data Communication Company (DCC) has been authorized by the Department of Energy and Climate Change (DECC) from Sept. 2013 to provide network operators with smart metering data from all customers [32]. DCC in turn grants a license to authorized third parties to provide services for the customers who have allowed them to use their data. Since this paper is based on the UK structure model, the DCCA has been considered in the proposed model to provide Local Transformer Agent (LTA) with the necessary data from their relevant individual Domestic Agents (DAs).

- Domestic Agent (DA): Each individual household has been considered as an autonomous agent who sends its related power consumption to DCCA and updates its status in receiving DCL from its relevant LTA.

- Local Transformer Agent (LTA): This agent acts as an aggregator which connects to number of DAs. The main target of LTA is to monitor, manage and control the power flow in its feeder while considering network constraints (power balance, network operating limit).

- DR Provider Agent (DRPA): This agent is connected to multiple LTAs in lower levels to define DCL as well as the duration of DR for each LTA.

\section{B. MAS Objectives}

In this study, DT operating status is considered as normal or emergency condition. Based on DT and power network constraints, emergency condition can occur due to two main reasons which in fact are the main objectives of the proposed MAS:

1- Load overloading of aggregated demand in each DT in which the LTA will make an attempt to reduce its total power demand by negotiating with its associated DAs.

Therefore, the objective of LTA can be calculated as:

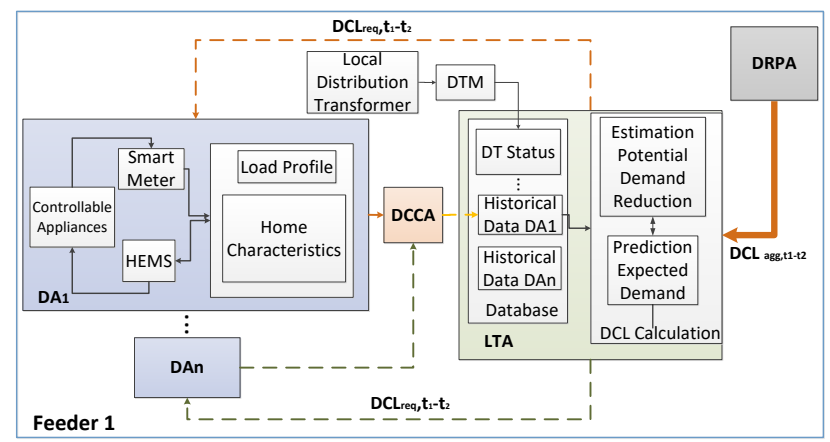

Fig. 1. Proposed MAS Framework

$$
\begin{array}{r}
\min \left(f=P_{T R A}=P_{D A, a g g}\right) \\
P_{D A, a g g}=\sum_{i=1}^{i=n} \sum_{j=1}^{j=p c} P_{D A}(i, j)(2)
\end{array}
$$

Constraint to:

$$
P_{T R A} \leq P_{T r a, c}
$$

Where,

PTRA: Instantaneous real power at LTA $(\mathrm{kW})$

PDA,agg: $\quad$ Aggregated Power of DAs

pc: $\quad$ Profile cluster

n: $\quad$ Number of households

$\mathrm{P}_{\mathrm{DA}}(\mathrm{i}, \mathrm{j})$ : Active power of $\mathrm{i}^{\text {th }}$ household in $\mathrm{j}^{\text {th }}$

PTRA,c: $\quad$ Loading Capability of LTA

2- Voltage drop of the distribution power network in which the network operator will send a DCL as a DR request to LTA in order to keep the total power/voltage of the network within the limit. Hence, the following constraint will add to the equations (3):

$$
P_{T R A} \leq D C L_{D R A}
$$

where DCLDRA denotes the amount of curtailed active power at each LTA.

\section{DR Algorithm and Agents Behaviuours}

Based on the overall proposed algorithm for DR control purpose, four different behaviours have been designed in order to determine the action/reaction of each agent toward other agents in pursuit the overall system and its own goals. The overall DR algorithm for booth TRA and DA has shown in Fig. 2, Fig. 3 and discussed in details the following.

1- UpdateData Behaviour : LTA constantly assess its status to ensure that it has enough capacity to handle the expected loads while waiting for any possible DR request from DR aggregator. Achieving these targets, LTA updates its information about its associated homes through DCCA in each time interval which is described as UpdateData Behaviour. Moreover, each DA sends its power consumptions to DCCA and updates its knowledge of a power distribution system in each time interval.

2- DRResponderBehaviour : In case of any emergency condition occurs, due to either load overloading of LTA or receiving DR request from DRPA, LTA clusters it's associated DAs to distinctive groups using historical load profiles and each household characteristics. Moreover, it predicts the expected demand and estimates the potential of demand responsiveness during DR event for each group of customers. The detailed process of calculation DR potential and clustering has been discussed further in section III and [10]. 
Then, LTA starts with the group of DAs with the highest probability of demand reduction potential and allocates appropriate DCL to them. It continues this allocation to the next group until it meets the overall objectives and maintains constraints of the power network. Afterwards, LTA sends an initial DR request containing DCL and DR duration as DRResponder Behaviour to selected DAs.

Receiving DCL from LTA, each DA calculates the current available demand reduction potential during DR and compares it with the DCL allocated to it. Then it replies to LTA by either accept or refuse the request.

3- DRRequester Behaviour : LTA allocates an update DCL to selected DAs based on receiving information from DAs and sends DR request to selected DAs as DRRequester behaviour .

4- RequestPerformer Behaviour: As soon as a DA receives a DCL, it performs the request in order to ensure that the total household power consumption does not exceed a given DCL during a DR event. Subsequently, a confirmation signal sends back by respective DA to inform TRA.

\section{METHODOLOGY}

This section presents the methodology for estimation the potential of demand responsiveness for each group of customer during DR event. It should be noted that a half hourly meter readings as well as an in-home survey of 3996 Irish households [9] have been applied for these analysis. The details process of the proposed methodology has been discussed in the following steps:

\section{A. Clustering domestic load profiles}

Considering aggregated domestic's loads, in first step, a number of customer classes have been made by K-means

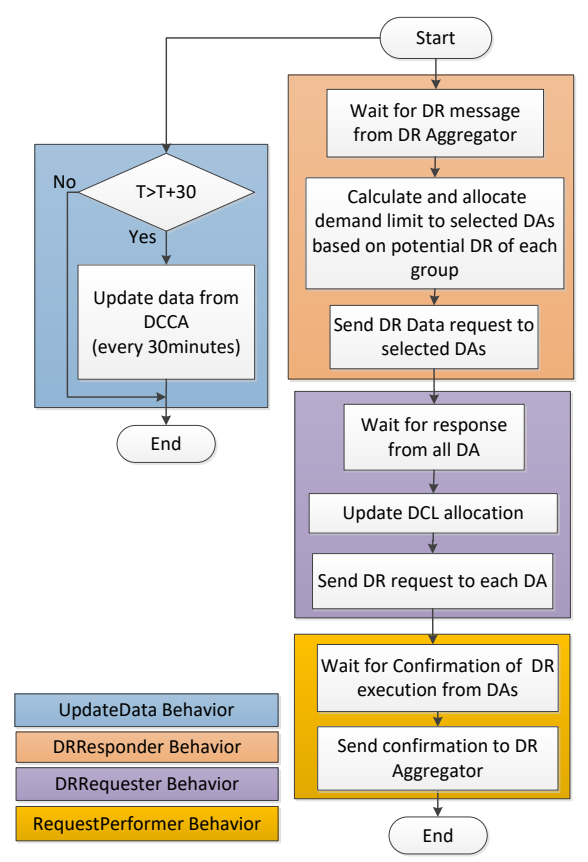

Fig. 2. Overall DR algorithm of a TRA resided

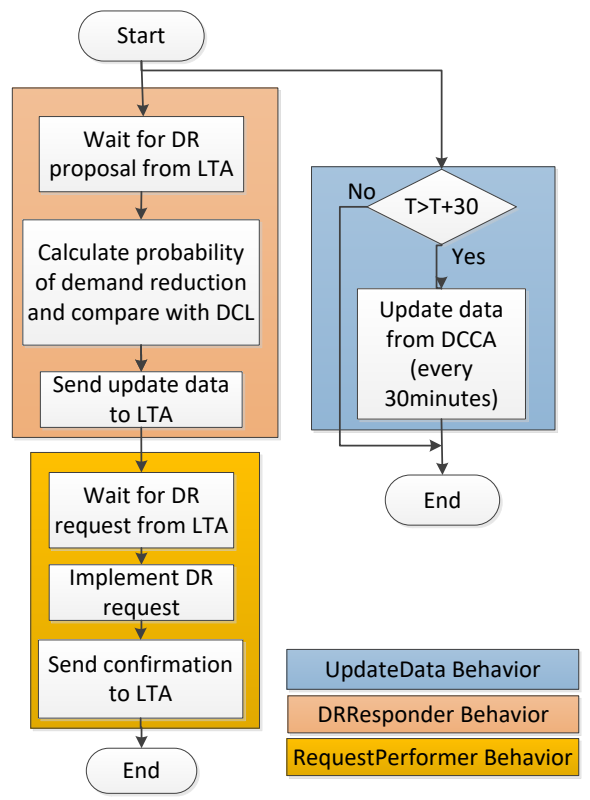

Fig. 3. Overall DR algorithm DA resided

clustering technique. This segmentation allows to group similar type of loads in terms of their electricity consumption. Accordingly, a mean aggregated load profile for each cluster $\left(P_{a g g, p c}\right)$ has been created using equation 1 :

$$
P_{a g g, p c}=\frac{\sum_{i=1}^{N d} P_{d, i}}{N d}
$$

Where $N d$ is the total day in the month and $P_{d, i}$ is the total daily energy consumption of $i^{t h}$ household in $d^{\text {th }}$ day of the month which is calculated by aggregating the power consumption of household $i$ at $\mathrm{t}^{\text {th }}$ timeslot in a typical day as:

$$
P_{d, i}=\sum_{t=1}^{48} P_{i, t}
$$

(6)The clustering has been applied for one summer month in July 2009 for weekdays. The result has been evaluated by Davies-Bouldin Index (DBI) and finally eight clusters have been selected. Fig. 4 shows the centroid of each created clusters.

\section{Charactrisation of clustered data}

Using in-home survey data, the households have been

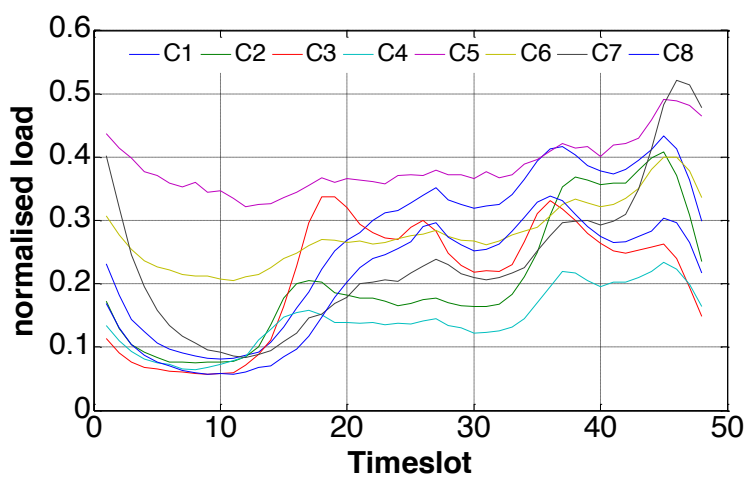

Fig.. 4. Centroid of 8 clusters of dataset 
clustered based on their interest in engagement to DR programs. The clustering results show three different groups of customers as highly motivated, less motivated and doubter. Then, only the households with higher willingness to participate in directly controlled demand in each resulting cluster have been selected. In this regard, the case study has been reduced to 3200 households. Details of each cluster of domestic loads have been presented in Table I and II. Moreover, the percentage of total occupants versus occupants during day, having children as well as wet appliances (washing machine, tumble dryer and dish washer) ownership for each cluster is presented.

\section{E. Estimating load reduction}

Having an understanding of customer's power consumption over time and using their characteristics, LTA can estimate the potential of demand curtailment from all aggregated loads within a group of customers $\left(P_{c, p c, a g g}\right)$ during DR event by using the following equations:

$$
\begin{gathered}
P_{c, p c, a g g}=\int_{t s}^{t e}\left(P_{h, \max , i, t}-P_{h, \min , i, t}\right) d t \\
P_{h, \text { max }, i, t}=\frac{\sum_{i=1, t=t s}^{i=t e} P_{h, \max , i, t}}{n, p c} \\
P_{h, \text { min }, i, t}=\frac{\sum_{i=1, t=t s}^{i=p c t=t e} P_{h, \text { min }, i, t}}{n, p c}
\end{gathered}
$$

where, $P_{h, \text { max }, i, t}$ and $P_{h, m i n, i, t}$ are the maximum and minimum power consumption of $\mathrm{i}^{\text {th }}$ household in cluster of $\mathrm{pc}^{\text {th }}$ type during DR event $\left(t_{s}-t_{e}\right)$ respectively which is obtained from similar-day- historical load profile.

Based on the achieved results, the priority of clusters based on DR potential during DR is listed in table III. As can be seen, there is a high relations between the number of households with high motivation in DR participation and priority clustering based on potential of demand curtailment. Households with children have experienced higher peak demand compare with other groups and therefore are more ideal for considering in DR programs. In addition, there is a similar distribution of wet appliances ownership for nearly all households.

\section{Modelling AND Simulation}

\section{A. $\quad$ Test Network}

A typical residential Low-Voltage (LV) network has been used as the test network (Physical layer) for the simulation using Matlab/Simulink. The network comprises of 39 households and is fed from a $11 / 0.415 \mathrm{kV}, 65 \mathrm{kVA}$ $\mathrm{MV} / \mathrm{LV}$ transformer. Properties of the test network have been summarized in Table IV. The one-line diagram of the test system has shown in Fig. 5.

The load profiles for each household have been created randomly with normal distribution around the

Table I.

CLUSTERS OF HOUSEHOLDS, BASED ON AVERAGE CONSUMPTION FOR WEEKDAYS IN JULY

\begin{tabular}{|c|c|c|c|c|c|c|}
\hline $\begin{array}{c}\text { Cluster } \\
\text { no. }\end{array}$ & $\begin{array}{c}\text { No. } \\
\text { households }\end{array}$ & $\begin{array}{c}\text { Household } \\
{[\%]}\end{array}$ & Consump. [kWh] & $\begin{array}{c}\text { Children } \\
{[\%]}\end{array}$ & $\begin{array}{c}\text { Occupants } \\
\text { during day } \\
{[\%]}\end{array}$ & $\begin{array}{c}\text { Wet } \\
\text { appliances } \\
\text { ownership [\%] }\end{array}$ \\
\hline 1 & 562 & 14.0 & 262.40 & 81 & 51 & 87 \\
\hline 2 & 648 & 16.2 & 202.16 & 75 & 38 & 89 \\
\hline 3 & 591 & 14.7 & 154.89 & 67 & 47 & 92 \\
\hline 4 & 392 & 9.8 & 104.63 & 45 & 83 & 82 \\
\hline 5 & 142 & 3.5 & 41.00 & 34 & 54 & 86 \\
\hline 6 & 394 & 9.8 & 135.58 & 67 & 27 & 88 \\
\hline 7 & 473 & 11.8 & 278.18 & 52 & 72 & 79 \\
\hline 8 & 794 & 19.8 & 292.75 & 90 & 30 & 95 \\
\hline
\end{tabular}

Table II.

SOCIAL SEGMENTATION OF THE CUSTOMERS

\begin{tabular}{|c|c|c|c|}
\hline \multirow{2}{*}{$\begin{array}{c}\text { Cluster } \\
\text { No }\end{array}$} & \multicolumn{3}{|c|}{ Attitude [\%] } \\
\cline { 2 - 4 } & $\begin{array}{c}\text { Highly } \\
\text { motivated }\end{array}$ & motivated & doubter \\
\hline 1 & 58.2 & 4.1 & 37.7 \\
\hline 2 & 31.8 & 6.4 & 61.8 \\
\hline 3 & 31.7 & 2.5 & 65.8 \\
\hline 4 & 19.8 & 2.8 & 77.4 \\
\hline 5 & 46.4 & 7.3 & 46.3 \\
\hline 6 & 41.9 & 9.5 & 48.6 \\
\hline 7 & 61.3 & 1.4 & 37.3 \\
\hline 8 & 49.3 & 4 & 46.7 \\
\hline
\end{tabular}

centroid of each cluster and keeping the same proportions of clusters as illustrated in table I. Fig. 6 shows the distribution of load profile in the test network.

B. MAS Simulation

The MAS structure (Virtual Layer) has been

Table III.

PRIORITY OF CLUSTERS BASED ON DR POTENTIAL

\begin{tabular}{|c|c|c|c|c|c|c|c|c|}
\hline $\begin{array}{c}\text { Class } \\
\text { no. }\end{array}$ & 1 & 2 & 3 & 4 & 5 & 6 & 7 & 8 \\
\hline $\begin{array}{c}\text { Priority } \\
\text { no. }\end{array}$ & 1 & 5 & 3 & 4 & 8 & 7 & 6 & 2 \\
\hline
\end{tabular}


implemented using the Java Agent DEvelopment Framework (JADE) platform. The MACSimJX middleware has been used as a link between the physical and virtual platforms.

\section{Agents Communication}

The communication among agents in JADE is performed in accordance with Foundation for Intelligent Physical Agents Communication Language (FIPA ACL) standards. The messages also adhere strictly to the Agent Communication Language (ACL) standard [11]. According to overall DR algorithm and designed behaviours, the communications and messages have been described in the following.

Each agent updates its data through communication with DCCA every 30 minutes using request-inform messages. DRResponder behaviour sends a DRDataRequest the content of a Call For Proposal (CFP) message which contains initial DCL and DR event duration for each DA. Receiving CFP, DA sends back an Agree/Refuse message to LTA. In addition, DA calculates and sends a Proposal message according to its current demand reduction potential. Based on receiving initial DCL the

The TRA coordinates the process of curtailment and sends the updated DCL allocation signal to the selected DAs in a Proposal message format. Then it waits for confirmation signal from all of DAs as an Inform-done message. Fig. 7 shows the sequence diagram of the interactions between agents in the proposed MAS framework during a DR event.

\section{Case Study Description}

The case study assumes that a DR event has occurred as a result of stress conditions causing by an unanticipated contingency in a transmission system (e.g., loss of generation or loss of transmission line). Therefore, LTA receives a DR event signal (DCL agg,t1-t2) equal to $51 \mathrm{~kW}$ demand reduction ( for a 2.5 hrs. peak hour period between 16:30 and 19:00 from DRPA.

\section{SimUlation RESUlts AND DisCUSSIONS}

The simulation results for one weekday in July have shown in Fig. 8 for the DT power $(\mathrm{kW})$ with and without DR control. As can be seen, the aggregation of bus loads is kept below the maximum loading capacity of DT $(65 \mathrm{~kW})$ in all time periods.

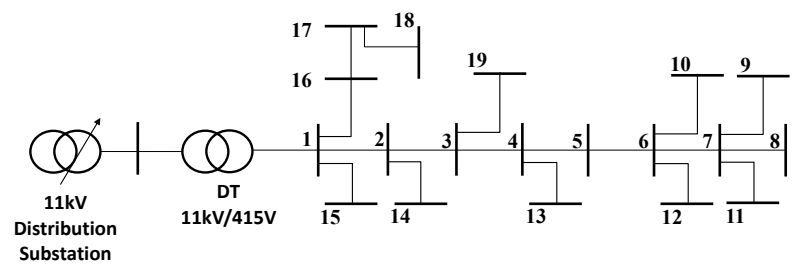

Fig. 5. One-line diagram of the simulation test network
After TRA receives a DCL $(51 \mathrm{~kW})$ from DRPA at 16:00 hrs., it checks its operating status to ensure that the power does not exceed from DCL. At 16:30, the operating state of the DT changes from normal to emergency since the instantaneous power at the TRA $(59 \mathrm{~kW})$ is higher than the assigned DCL $(51 \mathrm{~kW})$.

TRA update its status and knowledge about DAs from DCCA in each timeslot during DR event (16:30, 17:00, 17:30, 18:00, 18:30 and 19:00). Then, it sends a specific and distinctive DCL as a DR request message to all selected groups of DAs based on the proposed algorithm. Voltage level of each bus with and without DR during DR event has been shown in Fig. 9 in per-unit. Fig. 10 shows the percentage of demand curtailment for different clusters of customers in each time-interval during DR event. When

Table IV. LINE PARAMETERS OF THE TEST NETWORK

\begin{tabular}{|c|c|c|c|c|c|c|c|}
\hline \multicolumn{2}{|c|}{ LINE } & \multirow{2}{*}{$\mathrm{R}(\Omega)$} & $\mathrm{X}(\Omega)$ & \multicolumn{2}{c|}{ LINE } & \multirow{2}{*}{$\mathrm{R}(\Omega)$} & $\mathrm{X}(\Omega)$ \\
\cline { 1 - 3 } $\begin{array}{c}\text { Form } \\
\text { bus }\end{array}$ & $\begin{array}{c}\text { To } \\
\text { bus }\end{array}$ & & & $\begin{array}{c}\text { Form } \\
\text { bus }\end{array}$ & To bus & & \\
\hline 1 & 2 & 0.0415 & 0.0145 & 6 & 10 & 1.3605 & 0.1357 \\
\hline 2 & 3 & 0.0424 & 0.0189 & 4 & 13 & 0.140 & 0.0140 \\
\hline 3 & 4 & 0.0444 & 0.0198 & 3 & 19 & .7763 & 0.0774 \\
\hline 4 & 5 & 0.0369 & 0.0165 & 2 & 14 & 0.5977 & 0.0596 \\
\hline 5 & 6 & 0.0520 & 0.0232 & 1 & 16 & 0.1423 & 0.0496 \\
\hline 6 & 7 & 0.0524 & 0.0234 & 16 & 17 & 0.0837 & 0.0292 \\
\hline 7 & 9 & 0.0005 & 0.0002 & 17 & 18 & 0.3123 & 0.0311 \\
\hline 7 & 8 & 0.2002 & 0.0199 & 1 & 15 & 0.0163 & 0.0062 \\
\hline 7 & 11 & 1.7340 & 0.01729 & \multicolumn{2}{|c|}{ DT reactance } & \multicolumn{2}{|c|}{0.0654} \\
\hline 6 & 11 & 0.2607 & 0.026 & \multicolumn{3}{|c|}{} \\
\hline
\end{tabular}

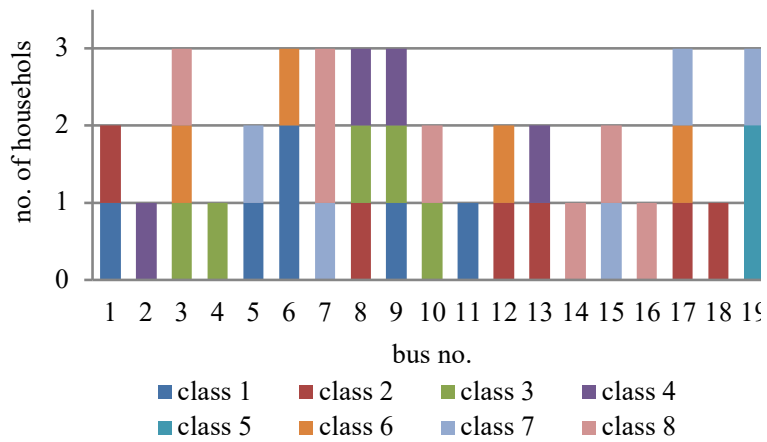

Fig. 6. Distribution of load profiles in the test network

higher power curtailment is needed more customers should involve in DR schemes. This indicates that aggregation of more customers can provide greater flexibility in terms of DR in order to improve the efficiency and reliability of the power system.

VI. CONCLUSION AND FUTURE RESEARCH 


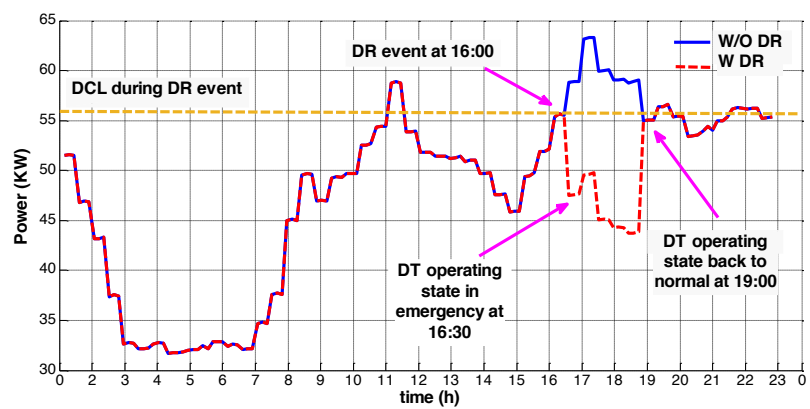

Fig. 8. Instantaneous Power (kW) of the DT during one week-day simulation in July with and without DR

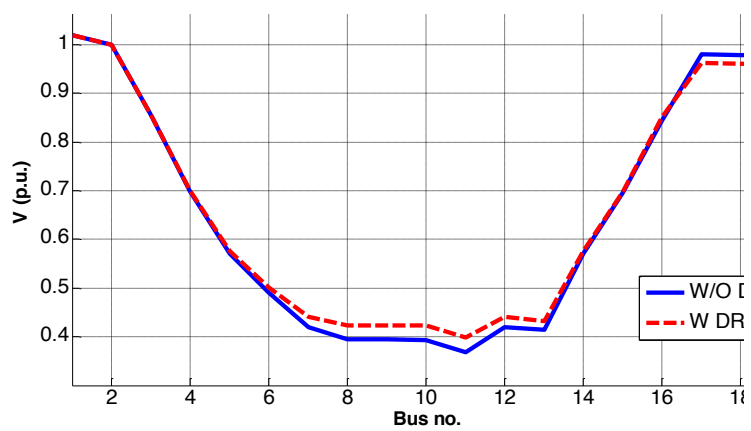

Fig. 9. Voltage (p.u.) of the DT in each bus during DR event

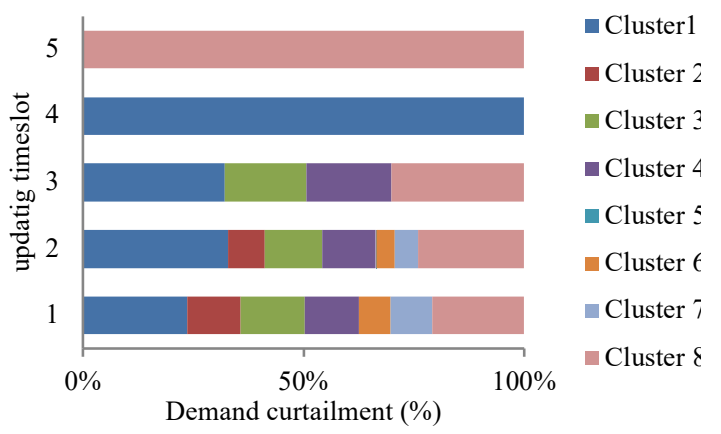

Fig. 10. Percentage of demand curtailment for each cluster in each time-interval during DR event

This paper proposes and develops a MAS framework to achieve flexible DR by controlling the power flow at local residential level. The proposed methodology is based on engagement as well as demand elasticity of households in DR programs considering various aspects of customer behaviour . A characterisation-based clustering for domestic loads has been applied in order to predict and assess the granularity of demand responsiveness for each group of customers. The simulation results show the effectiveness and capability of the proposed DR control scheme in controlling the voltage in feeders. That is achieved by maintaining smart distribution network active power within the limit in both normal and emergency conditions. The scalability of the proposed methodology allows integrating both centralized and decentralized voltage control and DR mechanism.
Future work includes implementing proposed MAS in a distribution network that consist of several feeders. Moreover, integrating price-based DR can be considered, taking in to account the price elasticity of different customers in each feeder. The communication structures such as different topologies and protocols as well as communication issues between agents are also a future interest.

\section{REFERENCES}

[1] J.Y. Park and J.K Park, "Real-time Volt/VAr control in a distribution system using multi-stage method," IFAC Symposium on Power Plants and Power Systems Control, Kananaskis, Canada, June 25-28, 2006.

[2] E. Bonabeau, "Agent-Based modeling: methods and techniques for simulating human systems," National Academy of Sciences, pp.7280-7287, 2002.

[3] M. Pipattanasomporn, M. Kuzlu, and S. Rahman, "An algorithm for intelligent home energy management and demand response analysis, " IEEE Transactions on Smart Grid, vol. 3, no. 4, pp. 2166-2173, 2012.

[4] J. Mathieu, M. Dyson, and D. Callaway, "Using residential electric loads for fast demand response: The potential resource and revenues, the costs, and policy recommendations," ACEEE Summer Study on Energy Efficiency in Buildings, pp. 189-203, 2012.

[5] A. Zakarianzadeh, O. Homaee, S. Jadid and P.Siano, "A new approach for real time voltage control using demand response in an automated distribution system, " Applied Energy, vol. 117, pp. 157-166, 2014.

[6] R. Fazel, J. Solanki and S.K. Solanki, "Demand response using multi-agent system," North American Power Symposium (NAPOS), Champaign, pp. 1-6, 2012.

[7] W. Khamphanchai, M. Pipattanasomporn, M. Kuzlu, J. Zhang, S. Rahman, " An approach for distribution transformer management with a multiagent system, "IEEE Transactions on Smart Grid, vol. 6, no. 3, pp. 1208-1218, 2015.

[8] A.N.M.M. Haque, P.H. Nguyen, T.H. Vo and F.W. Bliek, "Agent-based unified approach for thermal and voltage constraint management in LV distribution network," Electric Power Systems Research, vol. 143, pp. 462-473, 2017.

[9] Irish Social Science Data Archive. Data from the Commission for Energy Regulation (CER) - smart metering project. http://www.ucd.ie/issda/data/commissionforenergyreg ulationcer/; 2012 [accessed 27.02.17].

[10] S. Davarzani, I. Pisica and Gareth A. Taylor, "A novel methodology for predicting potential responsiveness in household demand, unpublished, presented at the Int. Conf. International Conference on Resilience of Transmission and Distribution Networks (RTDN), Birmingham, Birmingham, UK, Sept. 2017.

[11] 8. Vaucher, J., Ncho, A. "JADE Tutorial and Primer" http://www.iro.umontreal.ca/ vaucher/Agents/Jade/JadePrime r.html, [accessed 27.02.17]. accessed on 28/5/2005. 